\title{
Simple Ears Inspire Frequency Agility in an Engineered Acoustic Sensor System
}

\author{
José Guerreiro, Student Member, IEEE, Joseph C. Jackson, and James F.C. Windmill, Member, IEEE
}

\begin{abstract}
Standard microphones and ultrasonic devices are generally designed with a static and flat frequency response in order to address multiple acoustic applications. However, they may not be flexible or adaptable enough to deal with some requirements. For instance, when operated in noisy environments such devices may be vulnerable to wideband background noise which will require further signal processing techniques to remove it, generally relying on digital processor units. In this work, we consider if microphones and ultrasonic devices could be designed to be sensitive only at selected frequencies of interest, whilst also providing flexibility in order to adapt to different signals of interest and to deal with environmental demands. This research exploits the concept where the "transducer becomes part of the signal processing chain" by exploring feedback processes between mechanical and electrical mechanisms that together can enhance peripheral sound processing. This capability is present within a biological acoustic system, namely in the ears of certain moths. That was used as the model of inspiration for a smart acoustic sensor system which provides dynamic adaptation of its frequency response with amplitude and time dependency according to the input signal of interest.
\end{abstract}

Index Terms - bio-inspired acoustic transducer; dynamic frequency adaptation; feedback control; embedded system; realtime signal-processing; moth auditory system; resonance frequency; prototyping.

\section{INTRODUCTION}

$\mathrm{T}_{\mathrm{i}}$ design sensors which can be sensitive to small signal intensities when masked by noise may require unconventional thinking. Biology can provide inspiration to solve current human problems allowing the engineering landscape to borrow ideas to design clever sensors and systems. Some examples of bio-inspiration include robotic arms moved with the thoughts of paralyzed people [1], cochlear implants designed to provide hearing capabilities to deaf people [2,3], prostheses with the potential to help subjects who suffered brain damage [4], artificial retinas for blind people [5], miniaturized digital cameras inspired by the visual system [6], among other examples of bio-inspiration $[7,8]$. Looking into the ear example, auditory systems exhibit clever mechanisms to retrieve and process acoustic information. For instance, mechanical amplification and features like sharp frequency selectivity, and amplification with nonlinear compressive gains, are a result of active processes which notably enhance hearing capabilities [9-

This research is funded by the European Research Council under the European Union's Seventh Framework Programme (FP/2007-2013) / ERC Grant Agreement n. [615030].
11]. Neuronal coding and processing also plays a role in the signal processing in the hearing, where sound is encoded and processed in a form of electrical signals which the brain can interpret to make sense of the acoustic information [12].

Due to its relatively reduced complexity, insect hearing has been used as a model to inspire novel designs of miniaturized microphones [13-15]. Working towards that, we introduced a novel concept of a frequency-agile system bio-inspired by the auditory system of a moth - Noctua pronuba [16]. This is a smart acoustic transducer concept which automatically adapts its natural frequency response according to the intensity of input sound signals, using an embedded system in the feedback control loop that allows automatic updates of the mechanical properties of the front-end transducer. This paper is organized as follows: Section II introduces some background context about moth hearing; Section III details the overall anatomy and architecture of the purpose-built system; Section IV describes the embedded system setup developed to obtain results in realtime; Section V presents the theoretical model and simulations used to support the concept of a frequency agile sensor system and also to validate the experimental results; Section VI highlights the results performed to characterize the purposebuilt sensor system and its overall response; and finally Section VII summarizes the main conclusions and future prospects towards the development of frequency agile acoustic sensors and systems.

\section{BACKGROUND BIOLOGY - THE MOTH HEARING SYSTEM}

Hearing is achieved due to interactions between mechanical and electrical processes inside the auditory system. Natural coupling between these two mechanisms has evolved to enhance and improve transduction and subsequent processing of acoustic signals [17]. Aiming to retrieve acoustic cues, sound is converted to reciprocal electrical signals. For instance, in tympanic-like hearing (e.g. in a moth), mechanical forces acting over a sound-wave sensitive system (the tympanic membranepassive mechanical structure) leads to the process of ion influxes in the stretch-sensitive cells (i.e. mechanoreceptor cells) attached to it. Mechanoreceptor cells (e.g. stereocilia) are the type of biological sensors that transduce external mechanical stimulus (i.e. sound) - and resultant motion, into electrical signals. Alternate displacements induced on the

José Guerreiro, Joseph C. Jackson and James F.C. Windmill are members of Centre for Ultrasonic Engineering, Electronic and Electrical Engineering Department, University of Strathclyde, Glasgow, Scotland, UK (e-mail: \{jose.guerreiro, joseph.jackson, james.windmill\}@strath.ac.uk). 
tympanic membrane (from sound transmission) cause consequent cycles of increasing and decreasing force on the stereocilia (sound transduction). On one hand, positive cycles will induce the ion channels to open, which will depolarize the cells by increasing their potentials. On the other hand, negative cycles will induce the channels to close. This process represents an on-off mechanism with similarity to a positive half-wave rectifier response with a consequent capacitive charging effect. The cellular membrane of the mechanoreceptor cells can be seen as a small capacitor which charges every time strain increases and discharges when it decreases $[18,19]$. Auditory mechanoreceptor cells possess synaptic contacts with auditory neurons. During this transduction process, auditory neurons will depolarize with electrical charge until their threshold is reached - at which point an action potential occurs as a result of this comparative process. Neurons depolarize and can fire at different threshold levels, forming patterns which may represent some particular feature of the input signal such as, its intensity level [20]. Spike-like electrical signals are then conducted along the auditory nerves towards upper level neuronal systems in the brain which interpret and consequently can feed back responses to enhance peripheral transduction and processing of acoustic signals in a sort of feedback loop mechanism, as illustrated in Fig. 1.

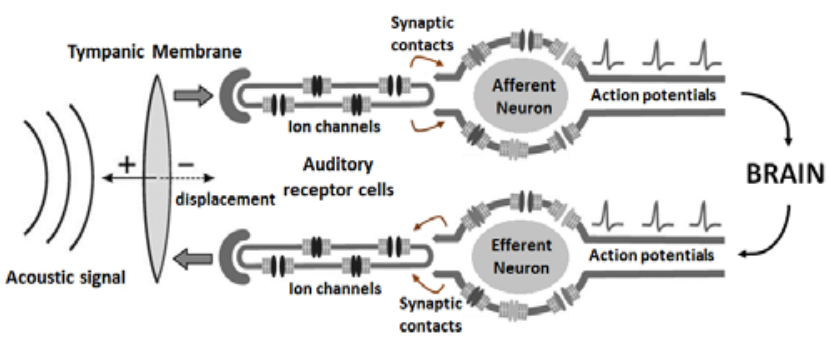

Fig. 1 - Overview of a tympanic-like auditory system (e.g. moth) showing transmission and transduction of acoustic signals into electrical information which is then processed and fed back by the brain to enhance peripheral conditioning and signal processing such as tuning. Redrawn and adapted from [21].

As previously mentioned, moths have a tympanum-like ear that acts as a pressure sensor for sound sensing. It has been reported in the literature that the moth auditory system is responsive to a wide band of frequencies - up to $300 \mathrm{kHz}$ [20]. In some Noctuid moths, it shows high sensitivity for quiet sounds at low frequencies (Fig. 2c-green trace; with resonance frequency $f_{0}$ ), and when stimulated with loud sounds, sensitivity is shifted to higher frequencies (Fig. 2c - orange trace; with resonance frequency $f_{0}{ }^{\prime \prime}$ ) instead. This auditory system automatically adapts its sensitivity towards higher frequencies in order to become frequency tuned with the hunting signals of bats [22]. Remarkably, with only one auditory receptor cell type (using two neurons with different potential thresholds) within the hearing organ, this moth is able to detect differences of sound intensity in order to cope with spectral changes within the signaling of bat echolocation calls. This adaptable mechanism of shifting towards higher frequencies is seen as a strategy of defense used to trigger behavioral reactions in order to avoid a fatal predation. This active tuning mechanism shows interesting features that can be exploited with great benefit to acoustic engineering as described throughout the following Sections.

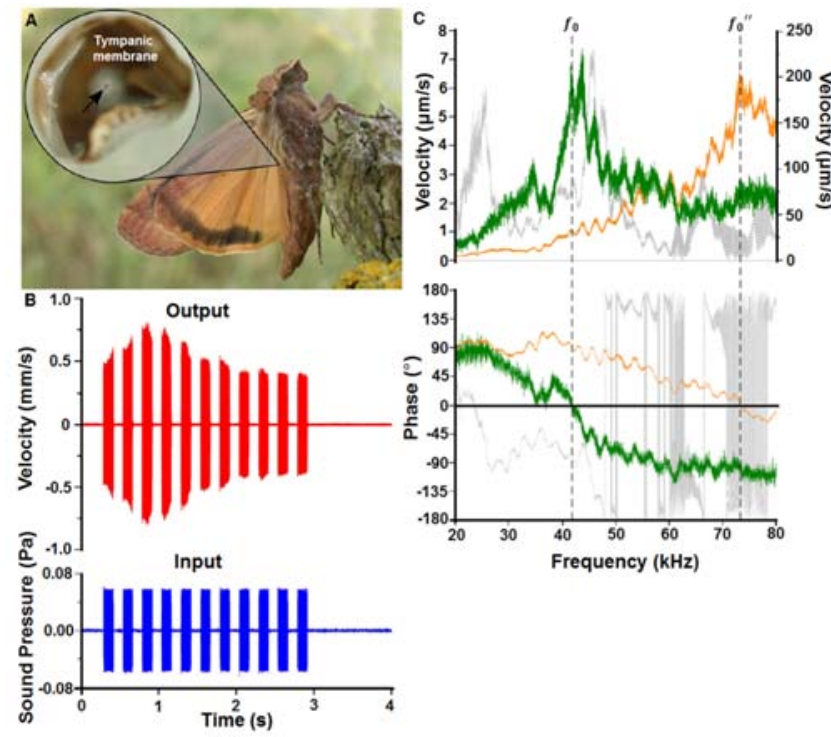

Fig. 2 - Tympanic membrane tuning responses in the moth Noctua pronuba. (A) Microscale ear of N. pronuba. (B) Mechanical response (red trace) of the tympanic membrane to bat-like incoming sounds (blue trace). (C) Frequency response of the tympanic membrane for low intensity stimulus (green trace) showing tuning at $f_{0}$, and for high intensity stimulus (orange trace) showing tuning at $f_{0}{ }^{\prime \prime}$. Redrawn and adapted with kind permission from [22].

\section{AnAtomy of a Frequency AgILE Acoustic System}

From the engineering point-of-view, the Noctuid moth hearing system can be represented as a system composed of an acoustic sensor with feedback computation capabilities as presented in Fig. 3, where the "Frequency Agile Sensor" block represents the tympanic membrane (i.e. front-end transducer); and the "Adapting Control System" block represents the backend neuronal processing (i.e. feedback computation) which activates the frequency agility of the front-end sensor to account for input signal stimuli.

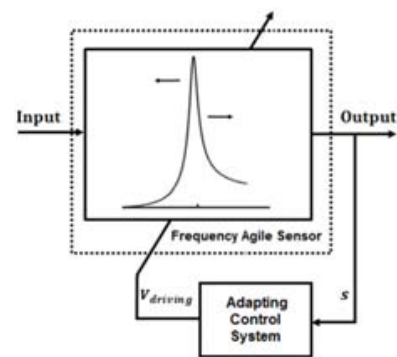

Fig. 3 - Diagram overview of the feedback control system used to implement the concept of a frequency agile transducer [16]. Where $s$ is the reference signal used to provide a processing response of the feedback system and $V_{\text {driving }}$ represents the control signal used to alter the frequency response of the front-end transducer.

\section{A. Frequency Agile Sensor}

For design purposes of this concept, the front-end transducer is made using a thin Kapton membrane placed and glued on top of a piezoelectric device (PZT stack) which is used to provide 
tension to the membrane, illustrated in Fig. 4. The PZT stack used is a PICMA $\AA$ stack multilayer piezo actuator $(\mathrm{P}-885.51)$ from PI Ceramic. Evaluation tests were conducted to analyze the frequency response of the transducer as detailed in Section VI.
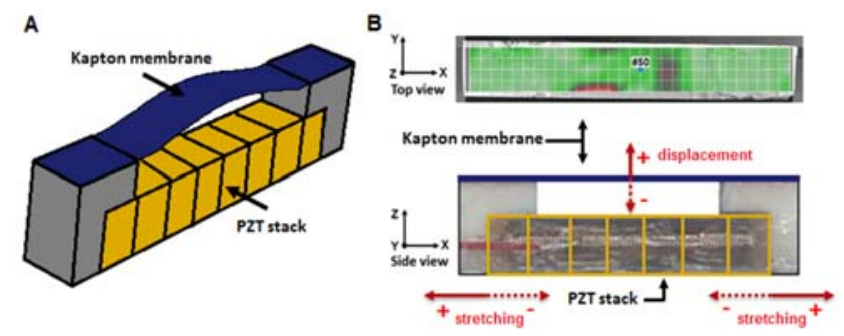

Fig. 4 - (A) 3D view of the acoustic transducer built with a Kapton membrane $(50 \mu \mathrm{m}$ thick) and a PZT stack (length $=18 \mathrm{~mm}$; height $=3 \mathrm{~mm}$; width $=3 \mathrm{~mm}$ ). (B) Top view of the transducer outlining a central point over the Kapton membrane (\#50). Side view of the transducer illustrating the polarity of displacement when the membrane is driven by sound and also the stretching direction which the PZT stack can provide to alter the behavior of the sensor.

\section{B. Adapting Control System}

In the moth hearing system, as reported in [22], the effective stiffness of the tympanic membrane might be physically dependent on the amplitude of the input stimuli reaching it (i.e. stiffness as a function of input amplitude). Following a similar approach, it is proposed that a back-end signal processing algorithm can be computed within a processing unit to progressively adapt the resonance frequency of the purposebuilt device. The algorithm is time and amplitude dependent and is designed to be easily executed while providing real-time results. The workflow of the signal processing is presented in Fig. 5, which mimics some "simple" mechanisms reported within auditory signal processing [12] such as, the first two blocks representing the "Mechanoreceptor cells role" and the following two blocks the "Neuronal cells role" - refer to Section II for further detail about each element.

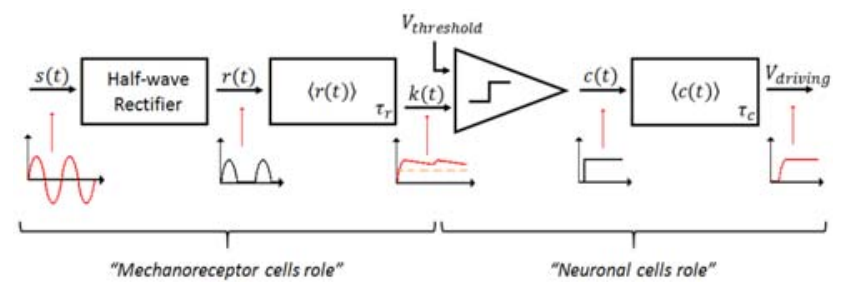

Fig. 5 - Schematic diagram of the "Adapting Control System" algorithm. Where $s$ represents the output signal of the system (recall Fig. 3) which is fed into the feedback pathway of signal processing; $r$ represents the halfwave rectifier output signal; $k$ shows the capacitive outcome from the mechanoreceptor cells charging effect; $c$ represents the on-off neuronal response (threshold dependent comparison) which is then smoothed $\left(V_{\text {driving }}\right)$ in order to provide a progressive control of the transducer's resonance frequency adaptation. $\tau_{r}$ and $\tau_{c}$ represent the time factors of $\langle r(t)\rangle$ and $\langle c(t)\rangle$ blocks, respectively.

The algorithm evolves as follows: the output signal $(s)$ readout from the sensor is averaged over time and once a predefined threshold is reached, the computational algorithm drives output signals $\left(V_{\text {driving }}\right)$ to control the front-end transducer's frequency response (e.g. stretching the membrane - changing stiffness). In this engineered version of "hearing" - the time averaging blocks $(\langle r(t)\rangle$ and $\langle c(t)\rangle)$ are implemented using first order IIR (Infinite Impulse Response) systems with transfer functions given in Z-domain by Eq. (1).

$$
H(z)=\alpha /\left(1-(1-\alpha) Z^{-1}\right)
$$

where $\alpha=1 / \tau$ represents the moving-average window coefficient and $\tau$ is the average time factor. Further details about the algorithm are provided in Section $\mathrm{V}$ when the theoretical model of the system is presented and its performance evaluated.

\section{EMBEDDED SYSTEM SETUP}

This Section describes the embedded system setup designed to implement the concept of the feedback control system illustrated in Fig. 3 that ensures results produced in real-time.

\section{A. Control and Processing Unit}

A schematic diagram of the embedded system setup used to dynamically adapt and control the mechanical properties of the transducer can be accessed in [16]. The acoustic transducer built with Kapton and PZT materials and the algorithm presented above were integrated in this setup. A laser vibrometer (head + controller: Polytec OFV 2700) providing an analog output voltage was used as the reference measurement signal of the displacements induced onto the membrane from acoustic stimuli. A 32-bit MCU (Micro-controller Unit) from STMicroelectronics (STM32F4) running at $168 \mathrm{MHz}$ is used to compute the algorithm. Pre-amplification and filtering are provided, integrated on a conditioning circuit for the output signals coming from the laser controller. Signal acquisition is done using an on-board 12-bit A/D converter with a sampling frequency of $50 \mathrm{kHz}$. Data is acquired and managed using interrupt-based routines. A threshold based algorithm is executed inside the CPU (Central Processing Unit) which is set according to the intensity of the input sound signals, such that the feedback control system can dynamically adapt the structural mechanics of the front-end transducer in real-time. The PZT stack is actuated using a 12-bit D/A converter with an additional analog driving circuit to amplify compatible output signals.

As previously described, the aim of this purpose-built embedded system is to implement the concept of a frequency agile acoustic sensor which provides results in real-time, so the setup should ensure real-time signal processing with accurate data sampling. The approach is based on hard and soft interrupt routines which allow prioritization of the acquisition task. The sampling rate of the $\mathrm{A} / \mathrm{D}$ required three hardware interrupt routines configured as follows: (1) Timer (configured in counter mode) is used to trigger the A/D to start acquisition; (2) $\mathrm{A} / \mathrm{D}$ end-of-conversion flag is used to signal the DMA controller to collect and transfer data to memory; (3) DMA data-transfer-complete flag is used to signal the CPU through a software interrupt routine when data is ready to be processed. The CPU computes the algorithm which sets the results through the $\mathrm{D} / \mathrm{A}$ converter that drives output signal. 


\section{B. Analog Conditioning Circuits}

As previously mentioned, the signals relating to the Kapton membrane's displacements are measured using an optical signal. Due to the inherent setup (e.g. manual alignment of the laser head + laser beam, etc.), the output signals given by the laser controller can exhibit low signal-to-noise ratio. In order to ensure suitable discrimination of signals acquired by the $A / D$, amplification and filtering are required. Amplification is performed using an instrumentation amplifier front-end with variable gain, up to $54 \mathrm{~dB}$, including an additional filter stage for low frequency suppression, with cutoff frequency approximately $2 \mathrm{kHz}$. Lowpass filtering is also provided using a Butterworth $4^{\text {th }}$ order filter, with cutoff frequency approximately $12 \mathrm{kHz}$, in a Sallen-Key configuration - this is for anti-aliasing filtering as well as high-frequency suppression. The final stage of this conditioning circuit is a variable DC level-shift circuit, which provides single-ended outputs to feed the $\mathrm{A} / \mathrm{D}$ (unipolar $0-3 \mathrm{~V}$ ) as well as to correct any DC-offset errors added from the previous stages of the circuit, as shown in the schematic of Fig. 6a. In order to operate the PZT stack within a voltage range up to $100 \mathrm{~V}$, it was necessary to design a circuit to amplify, up to $30 \mathrm{~dB}$ of gain, the output signals from the D/A (unipolar 0-3V). A non-inverter amplifier circuit using the PA78 device from Apex Microtechnology was used, as illustrated in Fig. 6b.

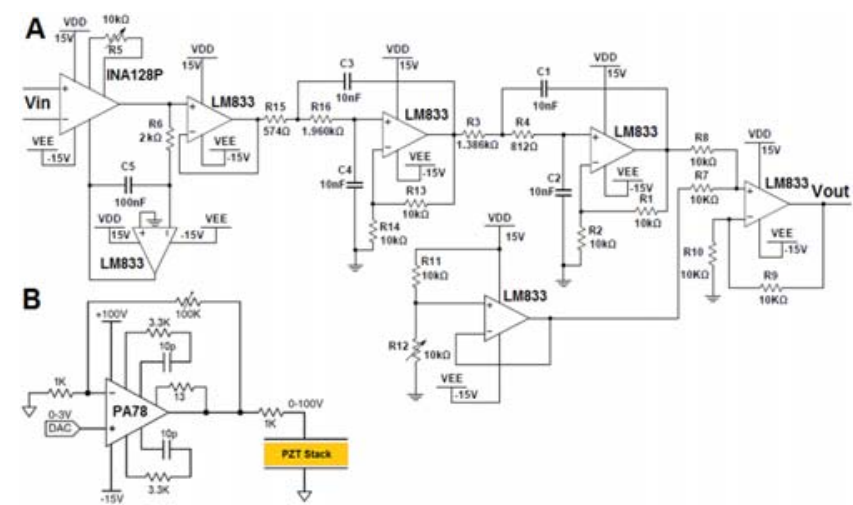

Fig. 6 - Schematic overview of the circuits used for conditioning and driving. (A) Conditioning circuit for the laser output signals - LNA, BPF and DC level shift; (B) Driver circuit used to amplify the output signals from the $\mathrm{D} / \mathrm{A}$ converter $(0-3 \mathrm{~V})$ to operate the PZT stack $(0-100 \mathrm{~V})$.

\section{THEORETICAL MODEL OF THE PURPOSE-BUILT SYSTEM}

This Section presents an overall theoretical model of the frequency agile system introduced in Section III.

\section{A. Front-end Transducer}

The structural acoustics and vibrational behavior of the frontend transducer obeys a linear single degree of freedom oscillator response, which for the purposes of this study can faithfully be expressed mathematically as a $2^{\text {nd }}$ order system with the Laplace expression presented in Eq. (2) - (refer to [23] for fundamental methods about structural acoustics and vibrations).

$$
H(s)=\frac{\omega_{0}^{2}}{s^{2}+s \frac{\omega_{0}}{Q}+\omega_{0}^{2}}
$$

where $\omega_{0}$ represents its natural resonance frequency; and $Q$ its quality factor. Theoretically, when providing additional tension over the membrane by pulling the structure apart (at the side points), it should change the effective stiffness and consequently alter its natural resonant frequency; since $\omega_{0}^{2}=$ $k / m$; $k$ - stiffness, $m$ - mass, as shown in Fig. 7 .
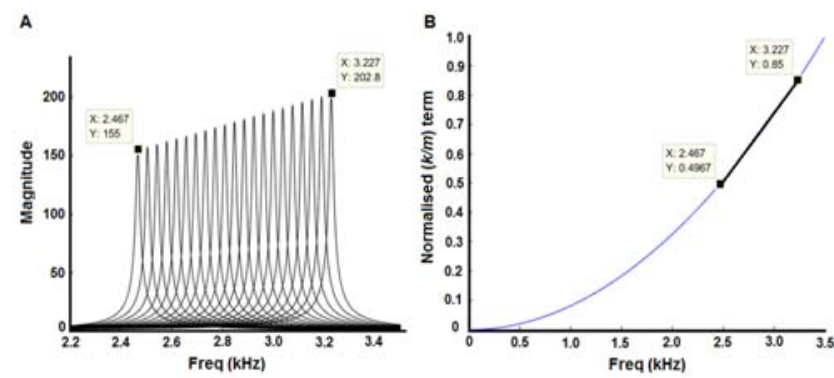

Fig. 7 - (A) Frequency response of a $2^{\text {nd }}$ order system while $\omega_{0}$ term is altered as a consequence of providing additional tension over the mechanical structure which consequently affect its stiffness - shifting frequency. (B) Graphical representation of $\omega_{0}^{2}=k / m$ - highlighting the region between two resonant frequencies, that shows a quasi-linear profile. Interval of frequencies highlighted are related to the experimental results presented in Section VI for comparison.

\section{B. Algorithm}

The "Adapting Control System" algorithm introduced in Fig. 5 is tested with synthetic sound signals as follows. The algorithm is divided in two parts: "Mechanoreceptor cells role" and "Neuronal cells role". As reported in the literature [12], it has been demonstrated that when increasing frequency of input stimuli mechanoreceptor cells gradually alter their response from alternating mode (AC) to direct mode (DC) - that behavior is achieved by the coupling between the initial two blocks (half-wave rectifier + lowpass filter) of this computational algorithm. In Fig. 8 the top row illustrates four examples of $s(t)$ signals (i.e. reference signal of the system which is used to feed the workflow of signal processing), that while increasing its frequency show a gradual change from $\mathrm{AC}$ to DC mode of operation. That behavior is illustrated in the bottom row of Fig. 8 with the $k(t)$ signal plot. This changing gradient is dependent on $\langle r(t)\rangle$ time term $-\tau_{r}$.

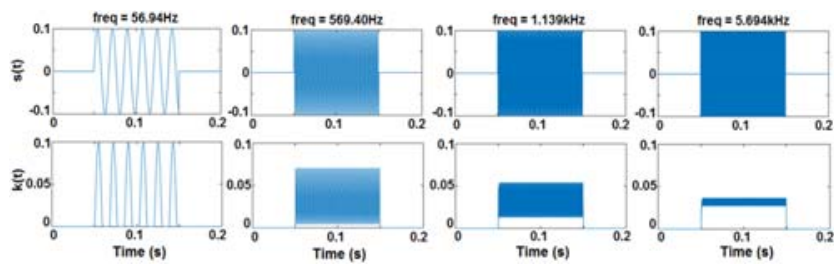

Fig. 8 - "Mechanoreceptor cells role" signal processing response showing gradual changing from $\mathrm{AC}$ to DC mode when frequency of input stimuli is increased - with $\tau_{r}=0.351 \mathrm{~ms}$.

The "Neuronal cells role" is the next step of the algorithm. It sets a comparison between a threshold previously defined with the resulted signal $k(t)$ followed by an additional lowpass filter - $\langle c(t)\rangle$. Fig. 9a presents three examples using different values of threshold (i.e. $0.01,0.025$ and 0.04 - from bottom to top). The output of the comparator is defined by $c(t)$ which is essentially an on-off output signal. This means that if $k(t)$ presents an oscillatory signature at the input of the comparator 
around its threshold value $\left(V_{\text {threshold }}\right)$ - Fig. 9a (top and middle plots) that will trigger an oscillatory on-off response of $c(t)$, as illustrated in Fig. $9 \mathrm{~b}-$ top and middle plots. So, the role that $\langle c(t)\rangle$ plays to stablish a more stable and smoothed output signal $\left(V_{\text {driving }}\right)$ of the algorithm is essential. On one hand, low averaging times within $\langle c(t)\rangle \rightarrow \tau_{c}$ will set faster overall responses (onset of the algorithm), however it will make the system less robust to fast switching at the comparator stage Fig. $9 \mathrm{~b}$ (top and middle plots on the left shows $c(t)$ signal with some ripple when $\tau_{c}=1 \mathrm{~ms}$ ). On the other hand, higher averaging times will increase the system's robustness against fast switching however it causes the system to react slower spending more time to achieve its steady-state, Fig. $9 \mathrm{~b}$ (top and middle plots on the right show $c(t)$ signal immune to fast switching but with slow convergence when $\tau_{c}=50 \mathrm{~ms}$ ). When the threshold is fully achieved with a DC component at $k(t)$ that will set a smoothed and progressive output response of the algorithm, as illustrated in Fig 9 - bottom plots when $V_{t h}=$ 0.01 . The overall time response of the algorithm is mainly dependent on $\tau_{c}$ since $\tau_{r}$ is kept significantly smaller in comparison in order to ensure DC modes of operation at low frequencies.

A
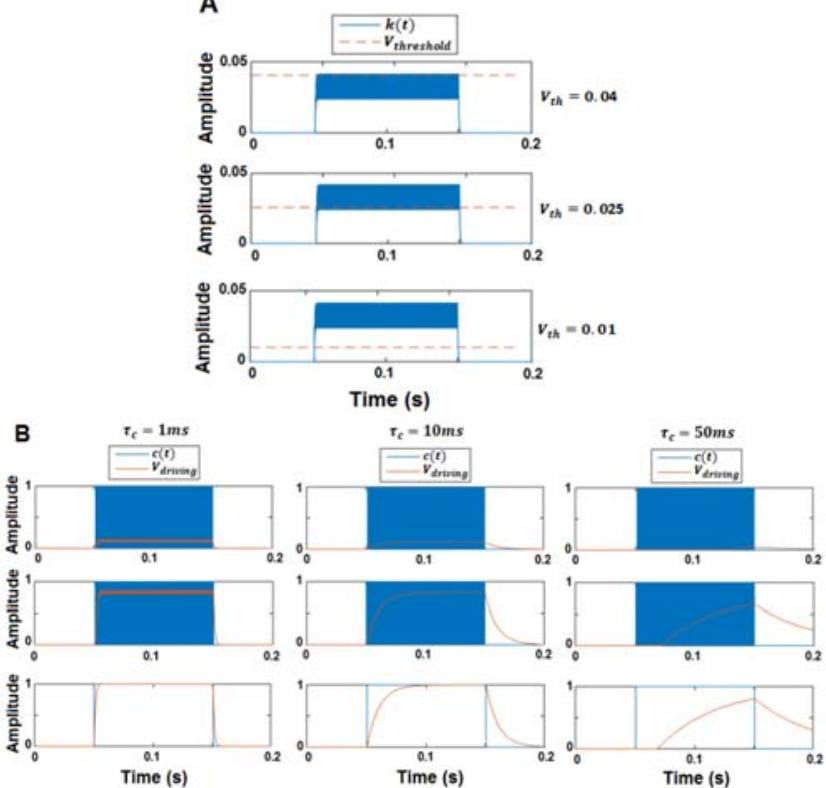

Fig. 9 - "Neuronal cells role" signal processing response showing threshold and time dependency settled mainly by the comparator and $\langle c(t)\rangle$ blocks, respectively. (A) shows three examples of $\mathrm{k}(\mathrm{t})$ signal using different thresholds; (B) illustrates the correspondent signals $\mathrm{c}(\mathrm{t})$ showing the influence of $\tau_{c}$ in the algorithm's performance and stability.

\section{Testing the Overall Feedback System Response}

In order to model the overall system response shown in Fig. 3 , three different synthetized sound signals are presented to the system's input to trigger frequency tuning that travels from $f_{0}$ towards $f_{0}^{\prime \prime}$, as illustrated in Fig. 10. First of all in (a), tuning is seen at the beginning of the input signal with frequency $f_{0}$, however once the algorithm starts to be iterated that will initiates its influence over the front-end sensor frequency response (i.e. modeling the mechanical properties of the frontend transducer being changed) and so after approximately $25 \mathrm{~ms}$ the system tuning becomes off-resonance. Secondly, in (b) the input signal with frequency $f_{0}{ }^{\prime}$ is not initially matched with the initial resonant frequency of the system $f_{0}$, however after approximately $25 \mathrm{~ms}$ of input stimulus being presented, the system shows resonant tuning matched with the input signal at frequency $f_{0}{ }^{\prime}$, due to feedback adaptation. Thirdly, in (c) the resonance tuning response of the system is matched with the input signal at frequency $f_{0}{ }^{\prime \prime}$. This happens when the algorithm reaches its steady-state of adaptation. If there is no acoustic input presented to the system, the threshold will not be reached and so the front-end sensor frequency response will return back to its initial resonance at $f_{0}$.

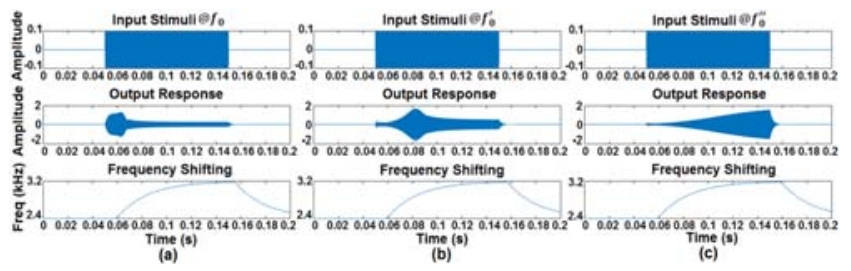

Fig. 10 - Modelling the feedback control system (illustrated in Fig. 3) showing three different resonance responses (a), (b) and (c) at $f_{0}=$ $2.467 \mathrm{kHz}, f_{0}^{\prime}=2.847 \mathrm{kHz}$ and $f_{0}^{\prime \prime}=3.227 \mathrm{kHz}$, respectively. Using $\tau_{r}=$ $0.351 \mathrm{~ms}, V_{\text {threshold }}=0.01$ and $\tau_{c}=25 \mathrm{~ms}$.

The impulse response of the overall system was also tested, presented in Fig. 11, and shows higher immunity to fast transients of the input signal when using higher values of $\tau_{c}$. So, the overall stability given by the feedback control system is dependent on those three parameters: $\tau_{r}, V_{\text {threshold }}$ and $\tau_{c}$. However, it can be mainly controlled by $\tau_{c}$ when kept higher than the settling time of the front-end sensor (which is $5 \mathrm{~ms}$, approximately), in detriment to the algorithm's time of convergence. For instance in Fig. $11 \mathrm{~b}-$ when $\tau_{c}=25 \mathrm{~ms}$, the algorithm accounts for the transducer's settling time to occur which then improves the system's stability when fast input transients appear, compared to when $\tau_{c}=1 \mathrm{~ms}$ as illustrated in Fig. 11a.

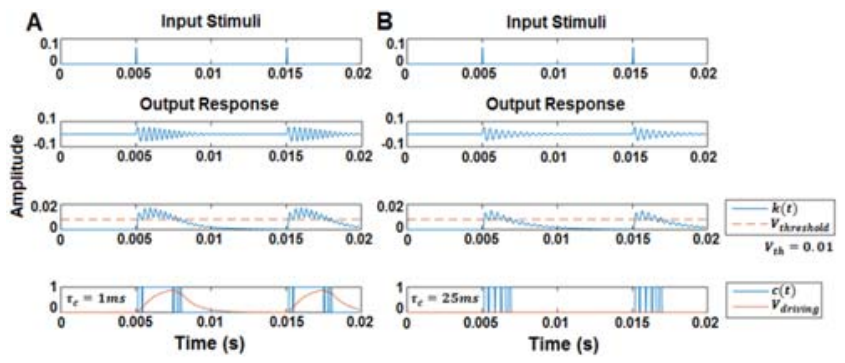

Fig. 11 - Impulse response of the overall feedback control system with settling time $\approx 5 \mathrm{~ms}-$ see "Output Response" plots; with $\tau_{r}=0.351 \mathrm{~ms}$ and $V_{\text {threshold }}=0.01$. (A) $\tau_{c}=1 \mathrm{~ms}$; and (B) $\tau_{c}=25 \mathrm{~ms}$.

\section{RESUlts}

This Section presents the results used to characterize and evaluate the front-end transducer as well as the overall embedded system response during real acoustic stimuli.

\section{A. Evaluation of the Mechanical Response of the Front-end Transducer}

Acousto-structural interactions in the transducer were evaluated using laser Doppler vibrometer (LDV) measurements as well as performing finite-element modelling in COMSOL. 
The frequency response resulting from COMSOL simulations is presented in Fig. 12a. It shows a suitable approximation of the resonance frequencies given by the transducer before its construction. It was important to have an approximation of their eigenvalue since the dimensions of the structure can play a significant role in that. The membrane was then measured using an LDV during acoustic stimuli and multiple resonance modes were identified, as presented in Fig. 12b.
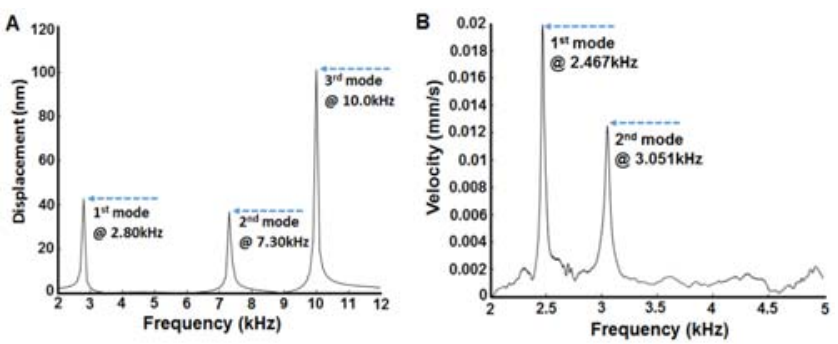

Fig. 12 - (A) COMSOL simulation showing the frequency response of the modeled transducer, presenting 3 resonant modes between $2-12 \mathrm{kHz}$. (B) Frequency response of the transducer measured with LDV, showing 2 resonant modes between 2 - 5kHz related to outlined central point (\#50) over the membrane (recall Fig. 4).

As previously described, the frequency response of the membrane can be altered by increasing tension over it and that was tested experimentally and is presented in Fig. 13a. The tension effect over the transducer was tested by driving the PZT stack with DC voltages between $0-100 \mathrm{~V}$ with $5 \mathrm{~V}$ step increments, resulting in a proportional increment of the natural resonant frequency of the sensor (Fig. 13b) - highlighting the $1^{\text {st }}$ resonant frequency being shifted from $2.467 \mathrm{kHz}$ to $3.227 \mathrm{kHz}(\Delta w=760 \mathrm{~Hz}$, where $\Delta w$ is the frequency shifting window that the transducer can be operated). This testing validates the transducer's design with the model presented in Section V, which we consider acceptable for the purposes of this work.
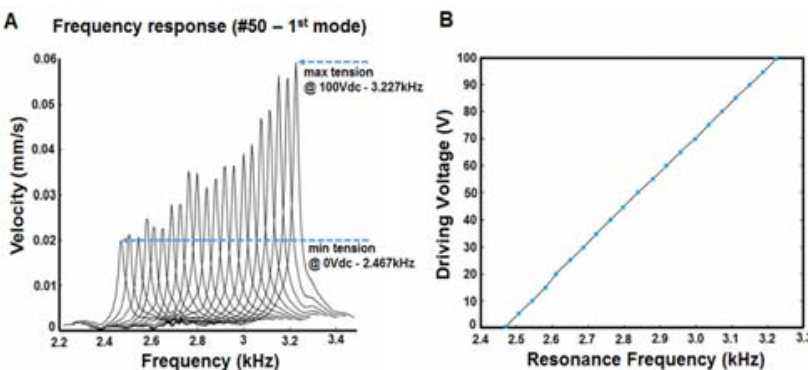

Fig. 13 - LDV measurements while providing DC voltages over the PZT terminals: (A) $1^{\text {st }}$ resonant mode of the Kapton membrane while providing different tensions (driving voltages); (B) Approximation to a quasi-linear shifting of the natural resonance frequency $-\Delta_{f} \approx 38 \mathrm{~Hz} @ \Delta_{V}=5 \mathrm{~V}$, that shows a favorable match with the theoretical model of the front-end transducer (recall Fig. 7 for comparison with the theoretical model).

\section{B. Tuning Response given by the Overall Embedded System}

The embedded system described in Section IV was tested integrating all its parts. The MCU was loaded with the algorithm following the processing workflow presented in Fig. 5. Fig. 14 shows three different tuning responses given by the presented embedded system. Frequency tuning travels from $f_{0}$ towards $f_{0}{ }^{\prime \prime}$, similar to that presented in the theoretical model in Section V - Fig. 10. First in Fig. 14(a), tuning is seen at the beginning of the input signal with frequency $f_{0}$, however once the membrane starts being stretched with a transverse positive tension, the mechanical properties of the membrane are changed and so is its frequency response - after approximately $25 \mathrm{~ms}$ the system tuning becomes off-resonance. Secondly in (b), the input signal with frequency $f_{0}{ }^{\prime}$ is not matched with the initial resonant frequency of the system $f_{0}$, however, after approximately $25 \mathrm{~ms}$ of the input stimulus being presented, and due to mechanical adaptations of the membrane, the system shows resonant tuning matched with the input signal at frequency $f_{0}{ }^{\prime}$. Thirdly in (c), the resonance tuning response of the system is matched with the input signal at frequency $f_{0}{ }^{\prime \prime}$ when the membrane is fully stretched (max. tension provided). If there is no acoustic input presented to the system, the algorithm will set no stretching output (min. tension provided) to the sensor which will return back to its initial resonance frequency at $f_{0}$.
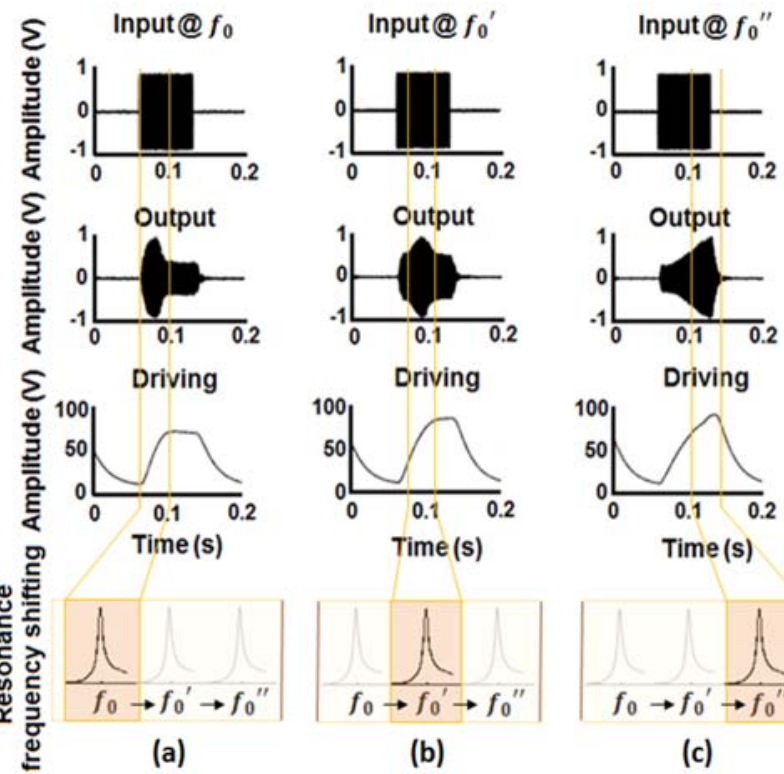

Driving

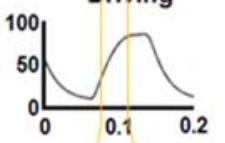

Driving
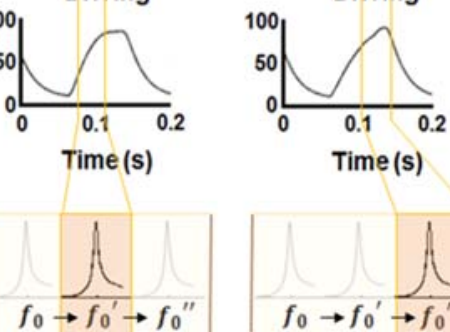

(b)

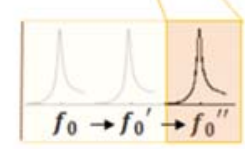

(c)

Fig. 14 - Results showing dynamic frequency adaptations given by the embedded system presenting time $\left(\tau=\tau_{r}+\tau_{c} \approx 25 \mathrm{~ms}\right)$ and amplitude $\left(V_{t h}=0.5 \mathrm{~V}\right)$ dependencies (recall Fig. 10 for comparison).

The processing time spent to compute the algorithm was measured at approximately $5 \mu$ s running the CPU at $168 \mathrm{MHz}$. The power consumption of the overall embedded system was estimated, at around $500 \mathrm{~mW}$ - based on the expected operating conditions of the CPU and digital hardware cores configured to implement the computational workflow of signal acquisition and processing as well as the analog circuitry used for signal conditioning and driving.

\section{CONCLUSIONS}

\section{A. Bio-inspired Acoustic Sensor System}

Engineering can look into biology to take inspiration. Over millions of years of evolutionary processes, nature has been developing ingenious sensors, allowing the conversion and processing of surrounding environmental information. A feedback mechanism acting over the tympanic membrane in 
Noctuid moth ears is used to achieve tuning [22]. Inspired by that, we introduced a novel engineered acoustic transducer concept combining mechanical signal processing with a complementary electrical feedback computation [16] - the transducer therefore computes, as the natural system does, to enhance peripheral signal conditioning and processing. This paper describes, models and validates the design of that acoustic sensor system, a combination of Kapton and piezoelectric ceramic materials. The use of materials such as PZT was fundamental to achieve the adaptations of this frontend transducer due to its mechano-electrical characteristics. Other smart materials such as polymers (e.g. EFOAM, PVDF or EAP) are also promising materials to exploit and use within new sensor-actuator designs in active control applications since they are light weight, flexible, low-cost, give easy integration into any size or material shape, among other characteristics [24]. Furthermore, a feedback computation was also developed in order to process acoustic signals based on "simple" mechanisms of auditory signal processing. Moreover, an embedded system setup was implemented in a feedback control loop to compute the algorithm that dynamically adapts the frequency response of the front-end transducer. Experimental results given by the purpose-built sensor system are acceptable and can be matched with their simulated model equivalents, which used to better describe and characterize the sensor system and its overall frequency agile behavior.

The purpose-built acoustic sensor system follows a quasilinear response over a functional frequency range of $760 \mathrm{~Hz}$. It has $38 \mathrm{~Hz}$ of frequency resolution at $5 \mathrm{~V}$, and its response time was set to be $25 \mathrm{~ms}$, which is the time defined for the sensor to operate from $f_{0}$ to $f_{0}{ }^{\prime \prime}$ using 0 to $100 \mathrm{~V}$, respectively. The system stability was evaluated in simulation and it is mainly controlled by the algorithm's time constant $\tau_{c}$. It is concluded that $\tau_{c}$ can ensure higher immunity to fast transients of the input signal if kept higher than the settling time of the front-end transducer itself. Accuracy, sensitivity and hysteresis, which are also fundamental specified requirements of standard sensing devices, are not quantified in this present study since it essentially targets the foundations of a novel concept that can potentially be exploited by sensor and system designers in the future development of acoustic devices, and the aim to present a final solution based on the purpose-built sensor and associated setup is out-of-the-scope of this paper.

\section{B. Future Work towards Frequency Agility in Acoustic Sensors}

It is not completely clear yet how the Noctuid moth hearing system tunes up [22]. However it is hypothesized that, either during the charging process mechanoreceptor cells may become stiffer (e.g. shortening) pulling somehow on the tympanic membrane - thus, once pulled the tympanic membrane is stretched and its mechanical structure and frequency response is altered; or it is due to feedback processing with efferent neurons controlling muscles attached to the tympanic membrane that plays a role acting on the structure instead (refer to Fig. 1 which illustrates a possible feedback loop using afferent and efferent neurons). Since the whole process which leads to this auditory adaptation response is still unclear, further investigations can be done from the biological point of view which consequently can also lead to further improvements of a frequency agile sensor system in the future.

Frequency agility can be either described as the function of a sensor system which cleverly adjusts its operating frequency to overcome the influence of interfering sources such as noise, or rather its adaptation to be tuned with desired signals of interest. This technique has already been exploited among radar and general radio applications to account for jamming or adverse atmospheric conditions [25]. Acoustic domains can also take advantage of this idea, as it has the potential to accomplish sound processing at the periphery of the sensor system by exploiting feedback coupling between mechanical and electrical processes at the transducer level (e.g. microphone point of view). The work described throughout this paper introduces the fundamentals of a novel concept of signal processing at the sensor level. An acoustic signal processing framework integrating a functional prototype system was engineered to support the concept of a frequency agile sensor, however further improvements may be included in order to address real world applications, outlined as follows:

(a) Front-end transducer: in this current study, a transducer made with a Kapton membrane $(50 \mu \mathrm{m}$ thick) glued on top of a PZT stack (length $=18 \mathrm{~mm}$; height $=3 \mathrm{~mm}$; width $=3 \mathrm{~mm}$ ) was used to sense acoustic signals, using an optical signal, and actuating with the piezoelectric functionality embedded in sensor itself. Further developments at the transducer level can be addressed to make the device smaller and easier to integrate by exploiting micro fabrication technology towards the design of miniaturised microphones and ultrasonic devices. Some ideas should be highlighted in this regard - (1) this concept of frequency agility is advantageous when using a high-Q transducer, so the design and fabrication requirements should accomplish that; (2) readout and actuation on the transducer can be done by using a combination of standard sensing and actuation techniques which can be optic (readout only), piezoelectric (readout and/or actuation) and capacitive (readout and/or actuation), with their associated demands for analog signal conditioning with respect to each one of those methods.

(b) Feedback computation: with proper adaptation, the signal computation can be designed to scan and hold at desired frequencies of interest instead of just moving up and down in the spectrum according to the intensity level of the input signal. Additional ideas can also be exploited in this regard are: (1) Other variables for decision making and adaptation can be used rather than only based on a static threshold. The threshold may be adaptable over time and this algorithm or any kind of computational technique chosen should evolve according to selected features of the input signal, for instance its amplitude and frequency signatures that if combined with the transducer's response may allow it to track multiband signals instead of single tone frequencies only; also, a combination of several agile sensors of this type placed in an array fashion can be exploited in this regard; (2) In real world applications a sensor that can adapt to environmental changes (atmospheric pressure, temperature, etc.), which can affect transducer's natural 
response, could have much improved performance. So, making a sensor adaptable over time might be of great importance to increase sensing fidelity in a measurement device system; (3) The algorithm presented in this paper exploits computational operations such as rectification, averaging and comparisons, which can be performed without the need for a digital computational unit executing DSP operations. Thus, the overall power consumption of a system might be reduced if exploring feedback between the front-end transducer and back-end computation using low power electronics based on analog signal processing techniques.

\section{ACKNOWLEDGEMENT}

The authors would like to thank the staff and researchers of Centre for Ultrasonic Engineering at University of Strathclyde for all the support throughout this research work.

\section{REFERENCES}

[1] B. Wodlinger, J. E. Downey, E. C. Tyler-Kabara, A. B. Schwartz, M. L. Boninger and J. L. Collinger. "Ten-dimensional anthropomorphic arm control in a human-brain machine interface: difficulties, solutions, and limitations". Journal of Neural Engineering, 12(1), 2014.

[2] B. S. Wilson and M. F. Dorman. "Cochlear implants: A remarkable past and a brilliant future". Hearing Research, 242(1-2): 3-21, 2008.

[3] M. Yip, R. Jin and H. H. Nakajima. "A Fully-Implantable Cochlear Implant SoC With Piezoelectric Middle-Ear Sensor and Arbitrary Waveform Neural Stimulation". IEEE Journal of Solid-State Circuits, 50(1): 214-229, 2015.

[4] T.W. Berger, M. Baudry, R.D. Brinton, J.-S. Liaw, V.Z. Marmarelis, B.J. Sheu and A.R. Tanguay. "Brain-implantable biomimetic electronics as the next era in neural prosthetics". Proceedings of the IEEE, 89(7): 993-1012, 2001.

[5] A. T. Chuang, C. E. Margo and P. B. Greenberg. "Retinal implants: a systematic review". Brithish Journal of Ophthalmology, 98(7): 852-856, 2014.

[6] Song Y.M., Malyarchuk V., Xiao J., Jung I., Choi K.J., Liu Z., Park H., Lu C., Kim R.H., Li R., Crozier K.B., Huang Y. and Rogers J.A., "Digital cameras with designs inspired by the arthropod eye". Nature, 497(7447): 95-99, 2013.

[7] Rahul Sarpeshkar. "Ultra Low Power Bioelectronics: Fundamentals, Biomedical Applications, and Bio-Inspired Systems". Cambridge University Press, 2010.

[8] Yoseph Bar-Cohen., "Biomimetics--using nature to inspire human innovation". Bioinspiration \& Biomimetics, 1(1): 1-12, 2006.

[9] A. J. Hudspeth. "Making an Effort to Listen: Mechanical Amplification in the Ear". Neuron, 59(4): 530-545, 2008

[10] Joseph C. Jackson and Daniel Robert, "Nonlinear auditory mechanism enhances female sounds for male mosquitoes". PNAS, 103(45): 16734 $16739,2006$.

[11] Joseph C. Jackson, James F. C. Windmill, Victoria G. Pook and Daniel Robert, "Synchrony through twice-frequency forcing for sensitive and selective auditory processing". PNAS, 106(25): 10177-10182, 2009.

[12] Schnupp, J., Nelken, I. and King, A., "Auditory Neuroscience: Making Sense of Sound". The MIT Press, 2012

[13] R.N. Miles and R.R. Hoy. "The Development of a Biologically-Inspired Directional Microphone for Hearing Aids". Audiology Neurotology, 11(2): 86-94, 2006

[14] Andrew Reid, James F.C. Windmill and Deepak Uttamchandani. "BioInspired Sound Localization Sensor with High Directional Sensitivity". Elsevier Procedia Engineering, 120: 289-293, 2015.

[15] Yansheng Zhang, Ralf Bauer, James F.C. Windmill and Deepak Uttamchandani. "Multi-Band Asymmetric Piezoelectric MEMS Microphone Inspired by the Ormia Ochracea". IEEE 29th International Conference on MEMS, 1114-1117, 2016.

[16] José Guerreiro, Joseph C. Jackson and James F.C. Windmill. "Bioinspired Frequency Agile Acoustic System". Proceedings IEEE Sensors Conference 2016.
[17] J. Hummel, S. Schöneich, M. Kössl, J. Scherberich, B. Hedwig, S. Prinz and M. Nowotny. "Gating of Acoustic Transducer Channels Is Shaped by Biomechanical Filter Processes". The Journal of Neuroscience, 36(8): 2377-2377, 2016.

[18] K. Jannis Hildebrandt, Jan Benda and R. Matthias Hennig. "Computational themes of peripheral processing in the auditory pathway of insects". Journal of Comparative Physiology. A Neuroethology, Sensory, Neural, and Behavioral Physiology, 201(1): 39-50, 2015.

[19] G. S. Pollack, A. C. Mason, A. N. Popper and R. R. Fay. "Insect Hearing". Springer, 2016.

[20] Moir, H.M., Jackson, J.C. and Windmill, J.F.C., "Extremely high frequency sensitivity in a simple ear". The Royal Society - Biology Letters, 9(4), 2013

[21] K. Fish, T. Schwalger, B. Linder, A.V.M. Herz and J. Benda. "Channel noise from both slow and adaptation currents and fast currents is required to explain spike-response variability in a sensory neuron". The journal of Neuroscience, 32(48):17332-17344, 2012.

[22] Windmill, J.F.C., Jackson, J.C., Tuck, E.L. and Robert, D., “Keeping up with bats: Dynamic auditory tuning in a moth". Current Biology, 16(24): 2418-2423, 2006

[23] Rossing, T. (Ed.) and Chaigne, A., "Chapter: 22 - Structural Acoustics and Vibrations". Handbook of Acoustics, $2^{\text {nd }}$ Edition. Springer, 2014.

[24] Windmill, J.F.C., Zorab, A., Bedwell, D.J., and Robert, D., "Nanomechanical and electrical characterization of a new cellular electret sensor-actuator". Nanotechnology, 19(3), 2008.

[25] Waters, W. M. and Linde, G.J., "Frequency-Agile Radar Signal Processing", IEEE Transactions on Aerospace and Electronic Systems, AES-15(3): 459-464, 1979.

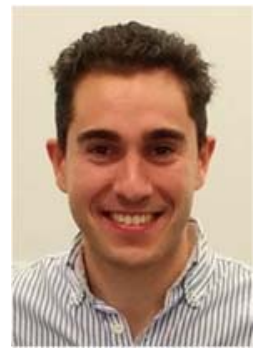

José Guerreiro (S'15) was born in Lisbon, Portugal, in 1988. He received the B.S. and M.S. degree in Electronic and Telecommunications Engineering from High Institute of Engineering of Lisbon in 2013, and currently is undertaking a Ph.D. degree in Electronic and Electrical Engineering at the University of Strathclyde, Glasgow, United Kingdom.

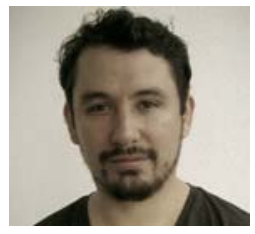

Joseph C. Jackson is a Lecturer in Electronic and Electrical Engineering at University of Strathclyde, based in the Centre for Ultrasonic Engineering. His research interests cover a wide range of subjects, such as the physical basis for hearing, sound production and reception in biology and engineering, and bio-inspired transducer and signal design.

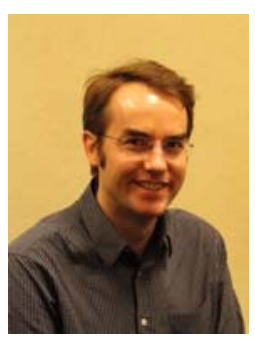

James F. C. Windmill (M'99) is a Reader in the Department of Electronic and Electrical Engineering at the University of Strathclyde, Glasgow, United Kingdom. $\mathrm{He}$ has over 18 years of research and development experience in the areas of sensors and hearing systems. His research interests are in the field of biologicallyinspired acoustic systems, from the fundamental biology to various engineering application topics. 